\title{
ON THE PROPERTIES OF SOME ADAPTIVE MORPHOLOGICAL FILTERS FOR SALT AND PEPPER NOISE REMOVAL
}

\author{
Marisol Mares JaVieR ${ }^{\bowtie, 1}$, CARlos Guillén Galván ${ }^{1}$, RAFAel LemuZ LópeZ² AND \\ JOHAN DEBAYLE ${ }^{3}$ \\ ${ }^{1}$ Facultad de Físico Matemáticas, Benémerita Universidad Autónoma de Puebla, ${ }^{2}$ Facultad de Ciencias de la \\ Computación, Benémerita Universidad Autónoma de Puebla, ${ }^{3}$ Mines Saint-Etienne, CNRS, UMR 5307, LGF, \\ Centre SPIN, 158 Cours Fauriel, 42023, Saint-Etienne Cedex 2, France \\ e-mail: marisol.maresja@alumno.buap.mx, cguillen@fcfm.buap.mx,rlemuz@cs.buap.mx, debayle@emse.fr \\ (Received May 27, 2020; revised January 16, 2021; accepted January 28, 2021)
}

\begin{abstract}
Mathematical Morphology (MM) is a tool that can be applied to many digital image processing tasks that include the reduction of impulsive or salt and pepper noise in grayscale images. The morphological filters used for this task are filters resulting from two basic operators: erosion and dilation. However, when the level of contamination of the image is higher, these filters tend to distort the image. In this work we propose a pair of operators with properties, that better adapt to impulsive noise than other classical morphological filters, it is demonstrated to be increasing idempotent morphological filters. Furthermore, the proposed pair turns out to be a $\wedge$-filter and a $\vee$-filter which allow to build morphological openings and closings. Finally, they are compared with other filters of the state-of-the-art such as: SMF, DBAIN, AMF and NAFSM, and have shown a better performance in time-quality ratio when the noise level is above $50 \%$.
\end{abstract}

Keywords: adaptive morphological filters, grayscale images, noise removal.

\section{INTRODUCTION}

Impulsive noise (or salt and pepper) is one of the most frequent types of noise present in digital images. This type of noise is caused by several factors such as faulty memory, scanning problems of the video sensors, decoding errors, transmission in a noisy channel, etc. The removing of this type of noise is one of the most important procedures that must be done before analyzing the characteristics of an image.

There exists several methods in the literature for the efficient removing of impulsive noise. One of the most used and robust non-linear filters is the standard median filter (SMF) (Huang et al., 1979), nevertheless, as noise increases, noise removing is less effective. Other algorithms like adapted median filter (AMF) (Ibrahim et al., 2008) and the Decision Based Algorithm (DBAIN) (Srinivasan and Ebenezer, 2007) tend to blur and distort the image. On the other hand, the Noise Adaptive Fuzzy Switching Median Filter (NAFSM) (Toh and Mat Isa, 2010) has better results than other median filters however it has a high computational complexity that makes it difficult to carry out. Furthermore, all these methods are focused only on the computational aspects rather than on properties of its filters.

In the framework of mathematical morphology, it is common to use combinations of erosions and dilatations for the removing of this type of noise (Maragos and Schafer, 1987; Song and Delp, 1990;
Oh and Chaparro, 1998; Mukhopadhysy and Chanda, 2002). Despite the fact that this kind of filters gives good results, as the level of degradation increases, they do not guarantee a good quality of the image. One of the reasons for its inefficiency is that they modify non-noisy pixels in an incorrect way contrary to most of the state-of-the-art filters. Added to this, the theoretical requirement of the property of adjunction between erosion and dilation (Serra, 1983; Debayle and Pinoli, 2005; Ćurić et al., 2014) makes it difficult to define morphological operators that do not modify non-noisy pixels. In the first section of this paper the aforementioned issues are analyzed in detail and illustrated, see figures 1,3 and 4 .

The main objetive of this work is to propose a family of real denoising morphological filters that overcome these difficulties for which it is demostrated that they turn out to be increasing and idempotent operators. Also, they are $\wedge$-filter and $\vee$-filter which allow to define mophological openings and closings. Finally it is shown that they are efficient for impulsive noise removal in comparison with other morphological filters and finally they are compared qualitatively and quantitatively to other filters from the literature.

The paper is organized as follows. In the first section, some concepts about classical and adaptive mathematical morphology are recalled. Besides the difficulty of defining morphological openings and 
closings that do not modify non-noisy pixels and at the same time effectively remove impulsive noise is analyzed. In the next section, it is proposed a pair of operators that overcome these dificulties and it is demonstrated to be $\wedge$-filter and $\vee$-filter (Serra and Vicent, 1992). Then, the proposed filter is evaluated and compared in terms of quality and execution time with some filters of the state of the art. Finally, in the last section, the conclusions are given.

\section{BACKGROUND ON MATHEMATICAL MORPHOLOGY}

A digital image can be described by the function

$$
f: \mathbb{Z}^{2} \rightarrow T
$$

where $T=\{0,1, \ldots, L\}$ and $\mathrm{L}$ is the maximum value of the intensity scale used. Let $(\mathscr{F}, \leq)$ be the lattice of functions with the order: $f \leq g \Leftrightarrow \forall x \in \mathbb{Z}^{2}, f(x) \leq$ $g(x)$. A noisy image of $f \in \mathscr{\mathscr { F }}$, is defined as $f_{r} \in \mathscr{\mathscr { F }}$ affected by impulsive noise where a fraction $p \in[0,1]$ of the points in the domain of $f$, which are randomly selected with uniform probability, change their value by the following composition,

$$
f_{r}(x)=\tau(\rho(1) \leq p, \tau(\rho(1) \leq 0.5,0, L), f(x))
$$

where $\tau\left(c, e_{v}, e_{f}\right)$ is the conditional function whose arguments are the logical condition $c$ to evaluate, the function to evaluate $e_{v}$ in case that $c=1$ (true) and the function to evaluate $e_{f}$ in case that $c=0$ (false). Also $\rho(1)$ is a number generating function with uniform distribution in the interval $(0,1)$. Note in the function $f_{r}$ that the values to assign are the maximum and the minimum of the gray scale.

\section{CLASSICAL MATHEMATICAL MORPHOLOGY}

Classical mathematical morphology studies images through series of operators that are, in a lot of cases, the result of the composition of two basic operations: erosion and dilation.

Definition 1 Erosion and dilation of a function $f \in \mathscr{F}$ through the structuring element $B \subset \mathbb{Z}^{2}$ are defined as:

$$
\varepsilon_{B}(f)(x)=\min _{y \in B_{x}} f(y) \text { and } \delta_{B}(f)(x)=\max _{y \in \hat{B}_{x}} f(y)
$$

respectively. Where $\hat{B}=\{-x: x \in B\}$ is the symmetric set of $B$, and $B_{x}$ is the translation of $B$ to point $x$.
One of the fundamental characteristics of this structuring element is that its size is much smaller than the domain of the image to be studied and thus, it acts as a probe or local analyzer.

The dilation and erosion are not inverse of each other and, in general, are not invertible. However, this allows defining another pair of operations based on their composition.

Definition 2 The opening and closing of $f$ by structuring element $B$ are defined as,

$$
\gamma_{B}(f)=\delta_{B} \varepsilon_{B}(f) \text { and } \varphi_{B}(f)=\varepsilon_{B} \delta_{B}(f) .
$$

respectively. The filters derived from the composition of the opening and the closing are the morphological operators used to eliminate noise in the grayscale images. Fig. 1 shows the result of applying filters $\gamma_{B} \varphi_{B} \gamma_{B}$ and $\varphi_{B} \gamma_{B} \varphi_{B}$ to Lena image contaminated with impulsive noise of $10 \%$ and $30 \%$, using the diamondshaped structuring element. It can be observed that the classic morphological filters are becoming less effective as the level of degradation increases, in addition to its softening effect that is not always something that one would expect to obtain. These problems are due in large part to the fact that the structuring element is the same at each point of the image, without distinguishing between noisy points and points that are important information of the original image.
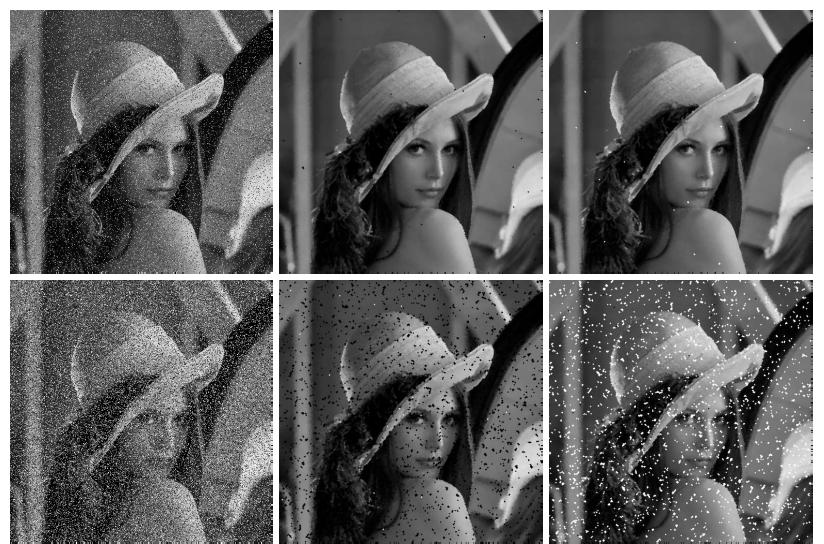

Fig. 1: From left to right. Up to down. first column: images with impulsive noise of $10 \%$ and $30 \%$; second column: morphological filter $\gamma_{B} \varphi_{B} \gamma_{B}$; third column: morphological filter $\varphi_{B} \gamma_{B} \varphi_{B} . B$ is the diamond-shaped structuring element.

Another filter used in MM for the removing of impulsive noise is the morphological center.

Definition 3 Being $\left\{\psi_{i}\right\}$ a family of operators over $\mathscr{F}$, meaning, $\psi_{i}: \mathscr{F} \rightarrow \mathscr{F}$. The morphological center is defined as

$$
C(f)=\min \left\{\max \left\{f, \min \psi_{i}(f)\right\}, \max \psi_{i}(f)\right\} .
$$



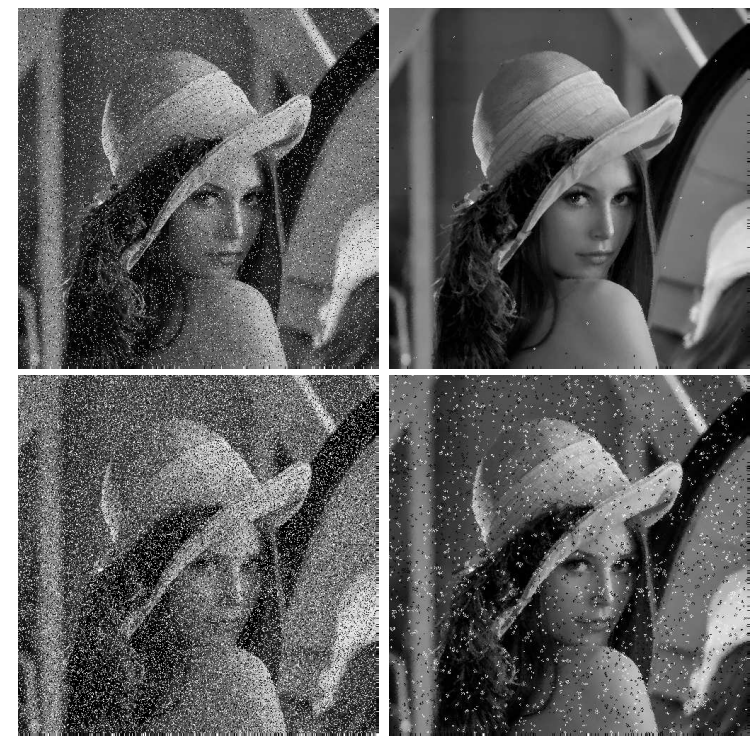

Fig. 2: . From left to right. Up to down. First column: images with impulsive noise of $10 \%$ and $30 \%$; second column: morphological center $C(f)$ where $B$ is the diamond-shaped structuring element.

Fig. 2 shows the result of applying the morphological center to the Lena image, with a noise level of $10 \%$ and $30 \%$, using the family of operators $\left\{\psi_{1}=\gamma \varphi \gamma, \psi_{2}=\varphi \gamma \varphi\right\}$. As can be seen, the noise not removed in the images filtered by morphological center is smaller than those in the images filtered by $\gamma \varphi \gamma$ or $\varphi \gamma \varphi$.

\section{ADAPTIVE MATHEMATICAL MORPHOLOGY}

The generalizations of the basic morphological operators (erosion and dilation) make up the area of mathematics known as adaptive mathematical morphology Debayle and Pinoli (2005;a; 2009); Angulo and Velasco-Forero (2011); Curić et al. (2014); Legaz et al. (2018). These generalizations are based on an essential property known as adjunction that is required to correctly define openings and closing from the combination of erosion and dilation. Let's define some important concepts.

Definition 4 Given $\Phi: \mathscr{F} \rightarrow \mathscr{F}$ an operator, it is said that:

1. $\Phi$ is increasing if for each pair $f, g \in \mathscr{F}$ so that $f \leq g$ then $\Phi(f) \leq \Phi(g)$.

2. $\Phi$ is idempotent if $\Phi^{2}=\Phi$.

3. $\Phi$ is extensive if for each $f \in \mathscr{F}, f \leq \Phi(f)$.

4. $\Phi$ is anti-extensive if for each $f \in \mathscr{F}, \Phi(f) \leq f$.

5. Si $\Phi$ is increasing, extensive and idempotent then $\Phi$ is a algebraic closing.
6. If $\Phi$ is increasing, anti-extensive and idempotent then $\Phi$ is an algebraic opening.

7. $\Phi$ is a morphological filter if it is increasing and idempotent on a lattice.

Definition 5 The operations $\varepsilon, \delta: \mathscr{F} \rightarrow \mathscr{F}$ form an adjunction on $\mathscr{F}$ if:

$$
\forall f, g \in \mathscr{F}: \delta(f) \leq g \Leftrightarrow f \leq \varepsilon(g) .
$$

The operations $\varepsilon_{B}$ and $\delta_{B}$ defined in (2) form an adjunction. On the other hand, in adaptive mathematical morphology the idea is to define a structuring element for each point in the domain of the digital image, which means, we define a family of structuring elements $Z(x)$, where $Z: \mathbb{Z}^{2} \rightarrow P\left(\mathbb{Z}^{2}\right)$ and $P\left(\mathbb{Z}^{2}\right)$ is the power set of $\mathbb{Z}^{2}$. Then we define the operations $\varepsilon_{Z}$ and $\delta_{Z}$ as:

$$
\varepsilon_{Z}(f)(x)=\min _{y \in Z(x)} f(y) \text { and } \delta_{Z}(f)(x)=\max _{y \in Z(x)} f(y) .
$$

In general, these operations are not morphological operations because the pair $\left(\varepsilon_{z}, \delta_{z}\right)$ does not always fulfill with the property of adjunction. However, given a family $Z(x)$ of subsets of $\mathbb{Z}^{2}$ so that $x \in Z(x)$, if for each $x$ we define

$$
\hat{Z}(x)=\left\{y \in \mathbb{Z}^{2}: x \in Z(y)\right\} .
$$

Then the pair $\left(\varepsilon_{z}, \delta_{\hat{Z}}\right)$ defines an adjunction. The set $\hat{Z}(x)$ can also be implicitly defined as:

$$
y \in Z(x) \Leftrightarrow x \in \hat{Z}(y)
$$

Moreover, one of the characteristics that makes many of the algorithms for the removing of impulsive noise efficient is that they preserve the pixels that have not been corrupted. Be $f_{r}$ a noisy image, to ensure that pixels that are not noisy are not modified when morphological operations are applied, we define a family of sets $\{Z(x)\}$ in such a way that:

$$
Z(x)=\hat{Z}(x)=\{x\} \text { if } x \in R^{c},
$$

where $R$ is a set that contains the noisy points and $R^{c}$ is the complement of $R$, that is, $R \cup R^{c}=\mathbb{Z}^{2}$. Let's see that the property stated in (6) cannot be combined conveniently with the condition stated in (5) to remove impulsive noise because the structuring element $\hat{Z}(x)$ from each noisy point it turns out to contain only noisy points. Indeed, let $x \in R$ such that $\hat{Z}(x) \cap R^{c} \neq \emptyset$ and let $y \in \hat{Z}(x) \cap R^{c}$, so $x \in Z(y)=\{y\}$ and therefore $x=y$, which is a contradiction. So that, $\hat{Z}(x) \subset R$ for every $x \in R$. Therefore, the dilation and the operations resulting from its combination with erosion, using a family of structuring elements that complies with the properties established in (5) and (6), will not eliminate 
the noise correctly. Since the morphological operations are not invertible, if we modify a non-noisy point, in general we will not be able to recover it. Therefore, adaptive morphological operators calculated using the reflected structuring element have disadvantages compared to other methods. Fig. 3 shows a magnified section of lena's image with some noisy pixels to which $\delta_{\hat{Z}}$ is applied. Here, $Z(x)=B_{x}$ is the diamondshaped structuring element for each noisy pixel, and $Z(x)=\{x\}$ for each non-noisy pixel. The structuring elements $Z(x)$ and the reflected structuring elements $\hat{Z}(x)$ for some pixels were drawn. As can be seen, each neighborhood $\hat{Z}(x)$ has only noisy pixels and the point $x$, so the resulting image contains more noisy pixels than original image. Further, the combination of this operation with others operations can also result in a noisier image, see figure 4 . In this work we study two operations with a special family of structuring elements that do not modify non-noisy pixels, and even that do not comply with the properties of extensivity and anti-extensivity, and therefore do not define morphological openings and closings, but they are increasing and idempotent, that is, morphological filters.

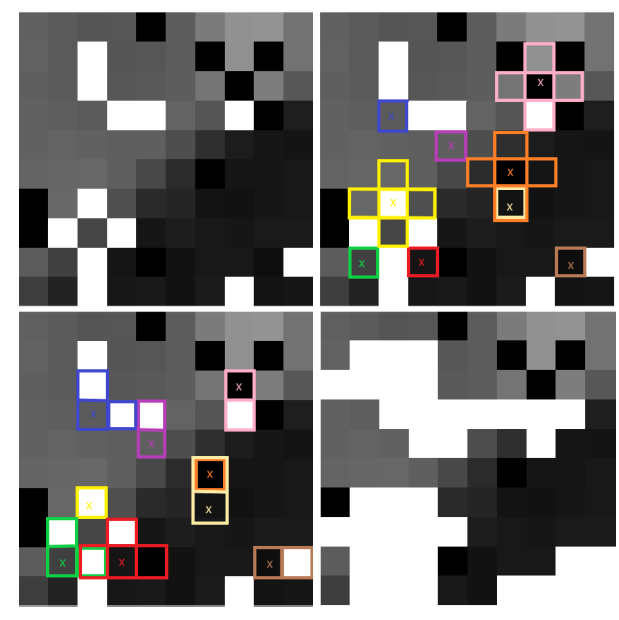

Fig. 3: From up to down. Left to right. first row: noisy image and estructuring elements $Z(x)$ for some pixels marked with an $x$. Second row: reflected estructuring elements $\hat{Z}(x)$ for some pixels marked with an $x$, and the resulting image $\delta_{\hat{Z}} f$.
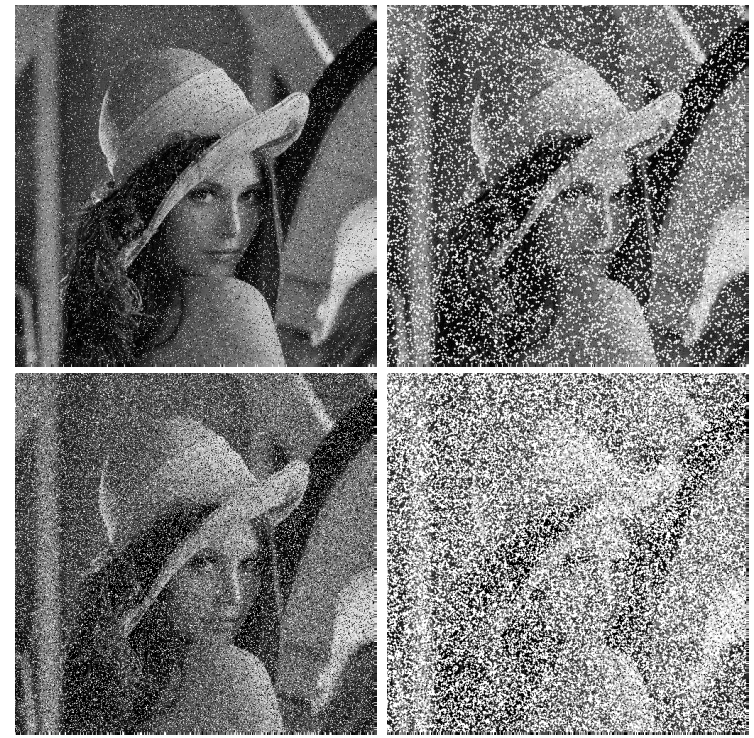

Fig. 4: From left to right. Up to down. first column: noisy image of $10 \%$ and $30 \%$; second column: $\varepsilon_{Z} \delta_{\hat{Z}} f$.

\section{PROPOSED ADAPTIVE MORPHOLOGICAL FILTER}

Considering $R, B \subseteq \mathbb{Z}^{2}$ so that $0 \in B$ and $B=\hat{B}$. For each $x \in \mathbb{Z}^{2}$ we define the function $Z_{R}: \mathbb{Z}^{2} \rightarrow P\left(\mathbb{Z}^{2}\right)$ as:

$$
Z_{R}(x)=\left\{\begin{array}{ccc}
\{x\} & \text { if } & x \in R^{c} \\
B_{x} & \text { if } & x \in R .
\end{array}\right.
$$

We define the operations $\varepsilon_{R}, \delta_{R}: \mathscr{F} \rightarrow \mathscr{F}$ as

$$
\varepsilon_{R} f(x)=\min _{y \in Z_{R}(x)} f(y) \text { and } \delta_{R} f(x)=\max _{y \in Z_{R}(x)} f(y),
$$

and the operations $\gamma_{R}, \varphi_{R}: \mathscr{F} \rightarrow \mathscr{F}$ as

$$
\gamma_{R} f(x)=\max _{y \in Z_{R}(x)} \min _{z \in Z_{R}(y)} f(z) \text { and } \varphi_{R} f(x)=\min _{y \in Z_{R}(x)} \max _{z \in Z_{R}(y)} f(z) .
$$

Note that $\gamma_{R} f=\delta_{R} \varepsilon_{R} f$ and $\varphi_{R} f=\varepsilon_{R} \delta_{R} f$.

Observation 1 If $x \in R$ and $y \in Z_{R}(x) \cap R$ then $x \in$ $Z_{R}(y)$. In fact, we have that $Z_{R}(x)=B_{x}, Z_{R}(y)=B_{y}$ and $y=x+w$ where $w \in B$, then $x=y+(-w)$ and $-w \in \hat{B}=B$, therefore $x \in B_{y}$.

Proposition 1 If $f \in \mathscr{F}$ and $x \in \mathbb{Z}^{2}$ then

$$
\varepsilon_{R} f(x) \leq \varepsilon_{R} \delta_{R} \varepsilon_{R} f(x)=\varepsilon_{R} \gamma_{R} f(x) .
$$


Proof

If $x \in R^{c}$ then $Z_{R}(x)=\{x\}$ and the inequality is trivial. Let $x \in R$. Developing the right side of (8) we have the following expression

$$
\varepsilon_{R} f(x) \leq \min _{y \in Z_{R}(x)} \gamma_{R} f(y) .
$$

This expression is equivalent to prove that $\varepsilon_{R} f(x)$ is lower bound of $\left\{\gamma_{R} f(y)\right\}$ where $y \in Z_{R}(x)$. That is,

$$
\forall y \in Z_{R}(x): \varepsilon_{R} f(x) \leq \gamma_{R} f(y) .
$$

a) If $y \in Z_{R}(x) \cap R^{c}$ then $y \in Z_{R}(x), \min _{z \in Z_{R}(x)} f(z) \leq$ $f(y)$ and $\gamma_{R} f(y)=f(y)$. Thus

$$
\varepsilon_{R} f(x)=\min _{z \in Z_{R}(x)} f(z) \leq f(y)=\gamma_{R} f(y) .
$$

b) If $y \in Z_{R}(x) \cap R$ then $x \in Z_{R}(y)$, see observation 1 . Hence,

$$
\varepsilon_{R} f(x) \leq \max _{z \in Z_{R}(y)} \varepsilon_{R} f(z)=\delta_{R} \varepsilon_{R} f(y)=\gamma_{R} f(y) . \square
$$

Proposition 2 Let $f, g \in \mathscr{F}$. The following properties are satisfied:

a) Increasing: $f \leq g \Rightarrow \gamma_{R} f \leq \gamma_{R} g$ and $\varphi_{R} f \leq \varphi_{R} g$.

b) Duality: $\gamma_{R} f=-\varphi_{R}(-f)$.

c) Idempotence: $\gamma_{R} \circ \gamma_{R}=\gamma_{R}$ and $\varphi_{R} \circ \varphi_{R}=\varphi_{R}$.

Proof

The properties a) and b) are directly derived from the definition of the operators $\gamma_{R}$ and $\varphi_{R}$. Then the proof for idempotence is presented. Let $x \in \mathbb{Z}^{2}$, if $x \in R^{c}$ then $Z_{R}(x)=\{x\}$ and $\gamma_{R} \circ \gamma_{R} f(x)=f(x)=\gamma_{R} f(x)$. Let's suppose that $x \in R$. By the proposition 1 and the increasing property of $\delta_{R}$ we have that

$\gamma_{R} f(x)=\delta_{R}\left(\varepsilon_{R} f\right)(x) \leq \delta_{R}\left(\varepsilon_{R} \delta_{R} \varepsilon_{R} f\right)(x)=\gamma_{R} \circ \gamma_{R} f(x)$.

Thus $\gamma_{R} f(x) \leq \gamma_{R} \circ \gamma_{R} f(x)$ for each $x \in \mathbb{Z}^{2}$. Now let us demonstrate that

$$
\gamma_{R} \circ \gamma_{R} f(x) \leq \gamma_{R} f(x)
$$

Since $Z_{R}(x)=\left(Z_{R}(x) \cap R\right) \cup\left(Z_{R}(x) \cap R^{c}\right)$, then:

$$
\begin{aligned}
\gamma_{R} \circ \gamma_{R} f(x) & =\max _{y \in Z_{R}(x)} \min _{z \in Z_{R}(y)} \gamma_{R} f(z) \\
& =\max \left\{\max _{y \in Z_{R}(x) \cap R} \min _{z \in Z_{R}(y)} \gamma_{R} f(z), \max _{y \in Z_{R}(x) \cap R^{c}} f(y)\right\},
\end{aligned}
$$

Let $y \in Z_{R}(x)$. If $y \in R$ then $x \in Z_{R}(y)$, see observation 1 . Then

$$
\min _{z \in Z_{R}(y)} \gamma_{R} f(z) \leq \gamma_{R} f(x) \Rightarrow \max _{y \in Z_{R}(x) \cap R} \min _{z \in Z_{R}(y)} \gamma_{R} f(z) \leq \gamma_{R} f(x) .
$$

On the other hand

$$
\begin{aligned}
\max _{y \in Z_{R}(x) \cap R^{c}} f(y) & \leq \max \left\{\max _{y \in Z_{R}(x) \cap R} \min _{z \in Z_{R}(y)} f(z), \max _{y \in Z_{R}(x) \cap R^{c}} f(y)\right\} \\
& =\gamma_{R} f(x) .
\end{aligned}
$$

Thus $\gamma_{R} \circ \gamma_{R} f(x) \leq \gamma_{R} f(x)$ for each $x \in \mathbb{Z}^{2}$. Finally, we have that $\gamma_{R} \circ \gamma_{R} f=\gamma_{R} f$. The property of duality and the idempotence of the function $\gamma_{Z}$ imply the following equalities:

$$
\begin{aligned}
\varphi_{R} f & =-\gamma_{R}(-f) \\
& =-\gamma_{R} \gamma_{R}(-f) \\
& =-\gamma_{R}\left(\gamma_{R}(-f)\right) \\
& =-\gamma_{R}\left(-\varphi_{R} f\right) \\
& =\varphi_{R} \circ \varphi_{R} f . \square
\end{aligned}
$$

The pair $\left(\varepsilon_{R}, \delta_{R}\right)$ does not define an adjunction, and consequently operations $\gamma_{R}$ and $\varphi_{R}$ are not extensive or anti-extensive then, they do not define morphological openings or closings. However, the operations $\gamma_{R}$ and $\varphi_{R}$ are idempotent, increasing and, therefore, define morphological filters. Next, we present a couple of filters built from $\gamma_{R}$ and $\varphi_{R}$ with additional properties.

Given $\psi, \Phi: \mathscr{F} \rightarrow \mathscr{F}$ two operators, we define the operators $\psi \wedge \Phi$ and $\psi \vee \Phi$ as

$(\psi \vee \Phi) f(x)=\psi f(x) \wedge \Phi f(x)=\min \{\psi f(x), \Phi f(x)\}$.

Definition 6 An operator $\Phi$ is a $\wedge$-filter if $\Phi(I \wedge \Phi)=$ $\Phi$, while $\Phi$ is $a \vee$-filter if $\Phi(I \vee \Phi)=\Phi$. If $\Phi$ is $a \wedge$ filter and $a \vee$-filter then $\Phi$ is a strong filter.

Proposition $3 \gamma_{R}$ is a $\wedge$-filter $y \varphi_{R}$ is $a \vee$-filter.

Proof

Let $f \in \mathscr{F}$. And $x \in \mathbb{Z}^{2}$, from observation 1 and proposition 1 we have the next equalities

$$
\begin{aligned}
\gamma_{R}\left(f \wedge \gamma_{R} f\right)(x) & =\max _{y \in Z_{R}(x)} \min _{z \in Z_{R}(y)}\left\{f(z) \wedge \gamma_{R} f(z)\right\} \\
& =\max _{y \in Z_{R}(x)}\left\{\min _{z \in Z_{R}(y)} \gamma_{R} f(z) \wedge \min _{z \in Z_{R}(y)} \gamma_{R} f(z)\right\} \\
& =\max _{y \in Z_{R}(x)}\left\{\varepsilon_{R} f(y) \wedge \gamma_{R} f(y)\right\} \\
& =\max _{y \in Z_{R}(x)} \varepsilon_{R} f(y) \\
& =\delta_{R} \varepsilon_{R} f(x) \\
& =\gamma_{R} f(x) .
\end{aligned}
$$


By duality property we have the next equalities

$$
\begin{aligned}
\varphi_{R}\left(f \vee \varphi_{R} f\right) & =-\gamma_{R}\left(-\left(f \vee \varphi_{R} f\right)\right) \\
& =-\gamma_{R}\left((-f) \wedge\left(-\varphi_{R} f\right)\right) \\
& =-\gamma_{R}\left((-f) \wedge \gamma_{R}(-f)\right) \\
& =-\gamma_{R}(-f) \\
& =\varphi_{R}(f) .
\end{aligned}
$$

The following proposition gives a sufficient condition to build openings and closings.

Proposition 4 Given $\Phi: \mathscr{F} \rightarrow \mathscr{F}$ an operator, if $\Phi$ is $\wedge$-filter, then $I \wedge \Phi$ is an opening while if $\Phi$ is $\vee$-filter then $I \vee \Phi$ is a closing (Serra and Vicent, 1992).

Because of proposition 4 we have that $I \wedge \gamma_{R}$ is a morphological opening and $I \vee \varphi_{R}$ is a morphological closing.

\section{PROPOSED FILTER}

In the case of impulsive noise, the noisy pixels are in the set $R=\left\{x: f_{r}(x)=0\right.$ or $\left.f_{r}(x)=L\right\}$, where $f_{r}$ is the noisy image. Next we present a result that will help us to have a simple interpretation of the filters $I \wedge \gamma_{R}$ and $I \vee \varphi_{R}$, and to reduce their computational cost when $R$ is defined as before.

Proposition 5 Let $f \in \mathscr{F}$, if $R=\{x: f(x)=$ 0 o $f(x)=L\}, R_{1}=\{x: f(x)=0\}$ and $R_{2}=\{x$ : $f(x)=L\}$. then $I \wedge \gamma_{R} f=\gamma_{R_{2}}(f)$ and $I \vee \varphi_{R}(f)=$ $\varphi_{R_{1}}(f)$.

Proof

Let $x \in \mathbb{Z}^{2}$, we have the following cases,

a If $f(x)=0$ then $I \wedge \gamma_{R} f(x)=f(x) \wedge \gamma_{R} f(x)=$ $f(x)=\gamma_{R_{2}} f(x)$.

$\mathrm{b}$ If $f(x)=L$ then $I \wedge \gamma_{R} f(x)=f(x) \wedge \gamma_{R} f(x)=$ $\gamma_{R} f(x)=\gamma_{R_{2}} f(x)$.

c $\quad$ In any other case $I \wedge \gamma_{R} f(x)=f(x)=\gamma_{R_{2}} f(x)$.

The proof that $I \vee \varphi_{R} f=\varphi_{R_{1}} f$ is like the previous one.

We propose to combine the pairs $\left(\gamma_{R_{2}}, \varphi_{R_{1}}\right)$ and $\left(\gamma_{R}, \varphi_{R}\right)$, by using the morphological center for the removing of impulsive noise as follows:

$$
C(f)=\min \left\{\max \left\{f, \min \psi_{i}(f)\right\}, \max \psi_{i}(f)\right\} .
$$

Where $\psi_{1}=\gamma_{R} \varphi_{R} \gamma_{R}$ and $\psi_{2}=\varphi_{R} \gamma_{R} \varphi_{R}$ or $\psi_{1}=$ $\gamma_{R_{2}} \varphi_{R_{1}} \gamma_{R_{2}}$ and $\psi_{2}=\varphi_{R_{1}} \gamma_{R_{2}} \varphi_{R_{1}}$. This filter has the advantage of cleaning an image more efficiently compared to the classic morphological center.

Next, the algorithm to calculate $\gamma_{R}$ is proposed. Note that the operation is divided into two parts, first calculated $\varepsilon_{R} f_{r}$ and then the composition $\gamma_{R}=$ $\delta_{R}\left(\varepsilon_{R} f_{r}\right)$, keeping the family of structuring elements fixed $Z_{R}(x)$ for both operations.

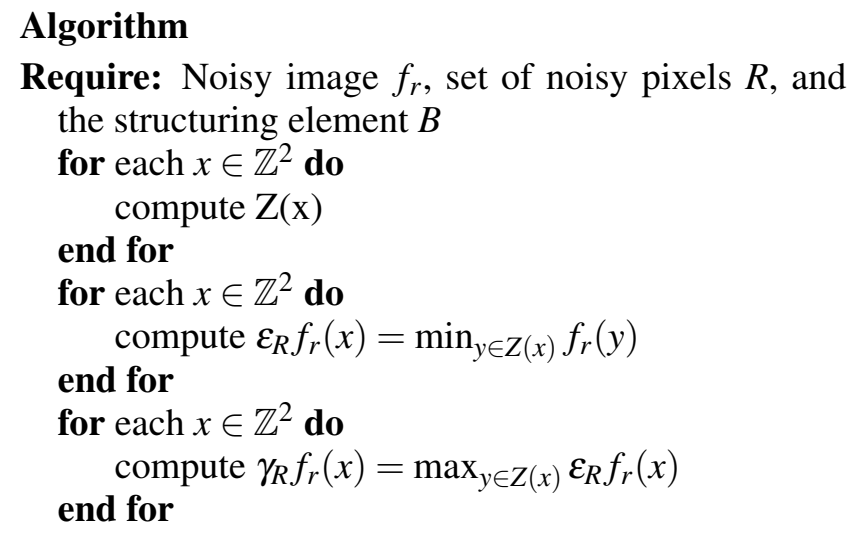

The algorithm to obtain $\varphi_{R}$ is similar. In the next section, the family used to compute the center is $\left(\gamma_{R}, \varphi_{R}\right)$, because this one has better results than the center computed with $\left(\gamma_{R_{2}}, \varphi_{R_{1}}\right)$.

\section{EXPERIMENTS AND RESULTS}

In this section the proposed method is evaluated and compared in terms of quality and execution time with other algorithms that include SMF, DBAIN, AMF and NAFSM, using the Lena's image of $510 \times 510$ size degraded with different noise levels ranging from $10 \%$ to $90 \%$ with increments of $10 \%$. The restoration quality of the image is measured with the structural similarity index (SSIM) (Wang et al., 2004), a metric that was designed to improve traditional metrics such as PSNR and MSE. The results of the evaluation are presented and compared in table 1 and figure 5. Table 3 shows the qualitative results of the application of these methods.

Lena's image in different sizes was used to compare the execution time of the different methods mentioned above, the results are presented in table 2. MATLAB 2017a on a PC equipped with 2.5-GHz CPU and $12 \mathrm{~GB}$ of RAM memory has been employed for the evaluation of computation time of all algorithms. 
Table 1: SSIM values for various filters applied on Lena's image at noise density levels from $10 \%$ to $90 \%$.

Filters

\begin{tabular}{clllll}
\cline { 2 - 6 } Noise $\%$ & SMF & \multicolumn{2}{c}{ DBAIN } & AMF & \multicolumn{2}{c}{ NAFSM Proposed } \\
\cline { 2 - 6 } 10 & 0.977 & 0.997 & 0.992 & 0.996 & 0.994 \\
20 & 0.929 & 0.993 & 0.987 & 0.991 & 0.988 \\
30 & 0.767 & 0.986 & 0.982 & 0.985 & 0.983 \\
40 & 0.492 & 0.976 & 0.973 & 0.975 & 0.975 \\
50 & 0.271 & 0.960 & 0.962 & 0.967 & 0.968 \\
60 & 0.142 & 0.936 & 0.947 & 0.953 & 0.959 \\
70 & 0.078 & 0.894 & 0.921 & 0.940 & 0.945 \\
80 & 0.041 & 0.827 & 0.878 & 0.914 & 0.921 \\
90 & 0.017 & 0.669 & 0.787 & 0.857 & 0.871
\end{tabular}

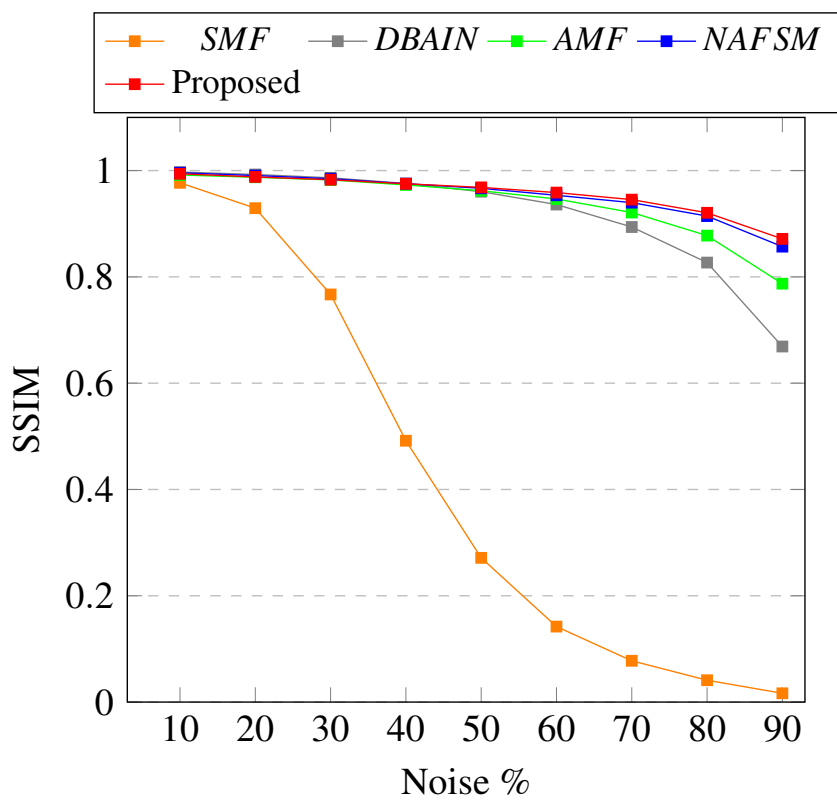

Fig. 5: Graph representing the SSIM values of table 1.

To study the robustness of our method, we considered a collection of 10 different images, Fig. 6 , which were contaminated with impulsive noise from $40 \%$ to $90 \%$ intensity and filtered with the 5 filters applied before. The results are presented in table 4 .

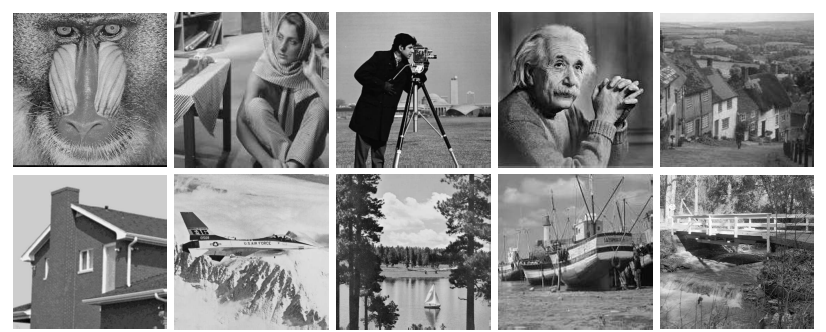

Fig. 6: Images. Up to down. From left to right. First row: Baboon, Barbara, Cameraman, Einstein and Hill; second row: House, Jet plane, Lake, Ship and Walk bridge.

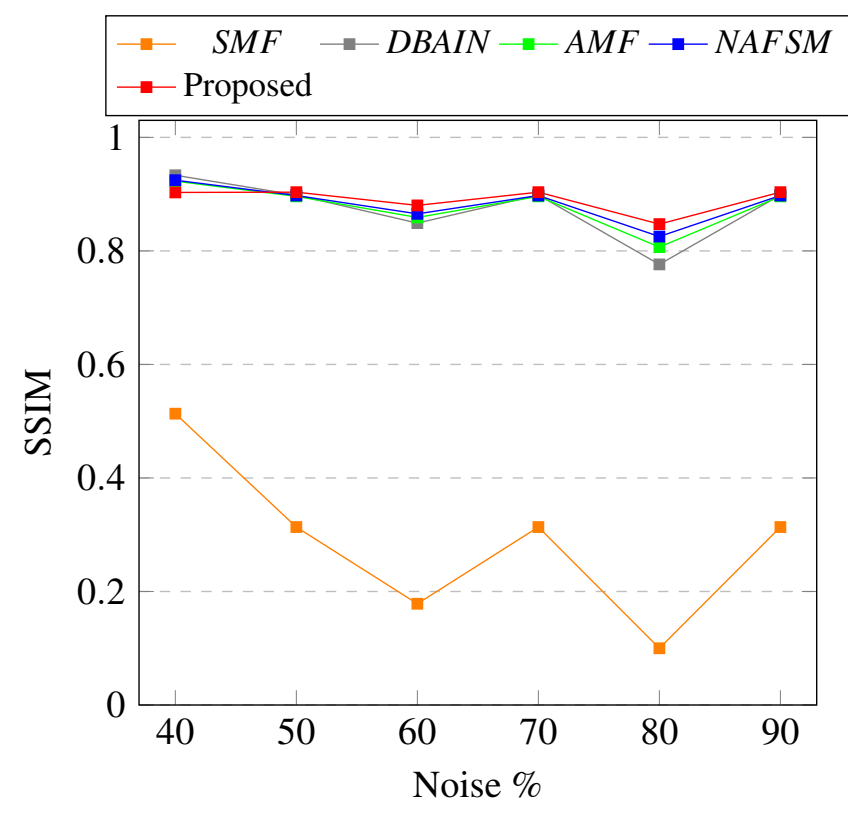

Fig. 7: Graph representing the SSIM average values of table 4.

\section{RESULTS}

As seen in figures 1,2 and 4 , the classic morphological filters are effective when the noise level is low, however, if the noise level increases the results of these filters are of low quality. From table 1 and Fig. 5 we can see that the proposed filter is effective and, unlike classical morphological methods, or adaptive, the filter remains stable by increasing noise levels. We also observed that when the noise level is above $50 \%$ the results of our filter are better compared to other state-of-the-art filters such as SMF, DBAIN, AMF and NAFSM.

From table 4 we can see that with images like those of Barbara and Einstein the NAFSM method has better results than the proposed method, and with images like walk bridge or jet plane the proposed method is better. Nevertheless, from noise level $50 \%$, in the average case our method was always the best, see figure 7 .

In table 2 it can be seen that both SMF method and proposed method have the best execution time. In addition, the proposed method also has the best results with regard to the restoration quality, therefore the proposed method has the best results with respect to the time-quality ratio. 
Table 2: Execution times for Lena image in different sizes contaminated with noise levels of $40 \%$ to $90 \%$. The best results were highlighted.

\begin{tabular}{|c|c|c|c|c|c|c|c|c|c|c|}
\hline Filters & SMF & DBAIN & $\mathrm{AMF}$ & NAFSM & Proposed & SMF & DBAIN & $\mathrm{AMF}$ & NAFSM & Proposed \\
\hline Size & \multicolumn{5}{|c|}{$40 \%$} & \multicolumn{5}{|c|}{$50 \%$} \\
\hline $128 \times 128$ & 0.000 & 0.267 & 0.037 & 0.371 & 0.019 & 0.000 & 0.268 & 0.034 & 0.450 & 0.019 \\
\hline $256 \times 256$ & 0.000 & 1.121 & 0.109 & 1.580 & 0.014 & 0.000 & 1.164 & 0.112 & 1.825 & 0.014 \\
\hline $348 \times 348$ & 0.001 & 2.066 & 0.259 & 2.852 & 0.018 & 0.001 & 2.066 & 0.231 & 3.607 & 0.018 \\
\hline $512 \times 512$ & 0.001 & 4.421 & 0.735 & 5.930 & 0.026 & 0.001 & 4.500 & 0.787 & 7.385 & 0.027 \\
\hline $640 \times 640$ & 0.001 & 7.015 & $\begin{array}{r}1.020 \\
60 \%\end{array}$ & 9.341 & 0.034 & 0.001 & 6.913 & $\begin{array}{l}1.217 \\
70 \%\end{array}$ & 11.799 & 0.036 \\
\hline $128 \times 128$ & 0.000 & 0.271 & 0.066 & 0.533 & 0.019 & 0.000 & 0.270 & 0.047 & 0.617 & 0.013 \\
\hline $256 \times 256$ & 0.000 & 1.176 & 0.133 & 2.175 & 0.015 & 0.000 & 1.101 & 0.190 & 2.521 & 0.014 \\
\hline $348 \times 348$ & 0.001 & 2.079 & 0.238 & 4.006 & 0.019 & 0.001 & 2.033 & 0.286 & 4.622 & 0.019 \\
\hline $512 \times 512$ & 0.001 & 4.401 & 0.936 & 10.174 & 0.028 & 0.001 & 4.442 & 0.838 & 10.047 & 0.029 \\
\hline $640 \times 640$ & 0.001 & 6.931 & $\begin{array}{r}1.254 \\
80 \%\end{array}$ & 14.109 & 0.036 & 0.001 & 6.915 & $\begin{array}{r}1.533 \\
90 \%\end{array}$ & 15.558 & 0.038 \\
\hline $128 \times 128$ & 0.000 & 0.273 & 0.065 & 0.710 & 0.017 & 0.000 & 0.273 & 0.190 & 0.807 & 0.012 \\
\hline $256 \times 256$ & 0.001 & 1.105 & 0.299 & 2.836 & 0.015 & 0.000 & 1.089 & 0.678 & 3.234 & 0.015 \\
\hline $348 \times 348$ & 0.001 & 2.074 & 0.475 & 5.276 & 0.019 & 0.001 & 2.073 & 1.232 & 5.952 & 0.020 \\
\hline $512 \times 512$ & 0.001 & 4.393 & 1.144 & 11.545 & 0.029 & 0.001 & 4.661 & 2.581 & 12.849 & 0.032 \\
\hline $640 \times 640$ & 0.001 & 6.982 & 2.022 & 18.186 & 0.040 & 0.001 & 6.898 & 3.995 & 20.047 & 0.044 \\
\hline
\end{tabular}

Table 3: From left to right: First column: Lena images at noise density levels of 50\% to $90 \%$;; second column: SMF; third column: DBAIN; fourth column: AMF; fifth column: NAFSM and the proposed method.

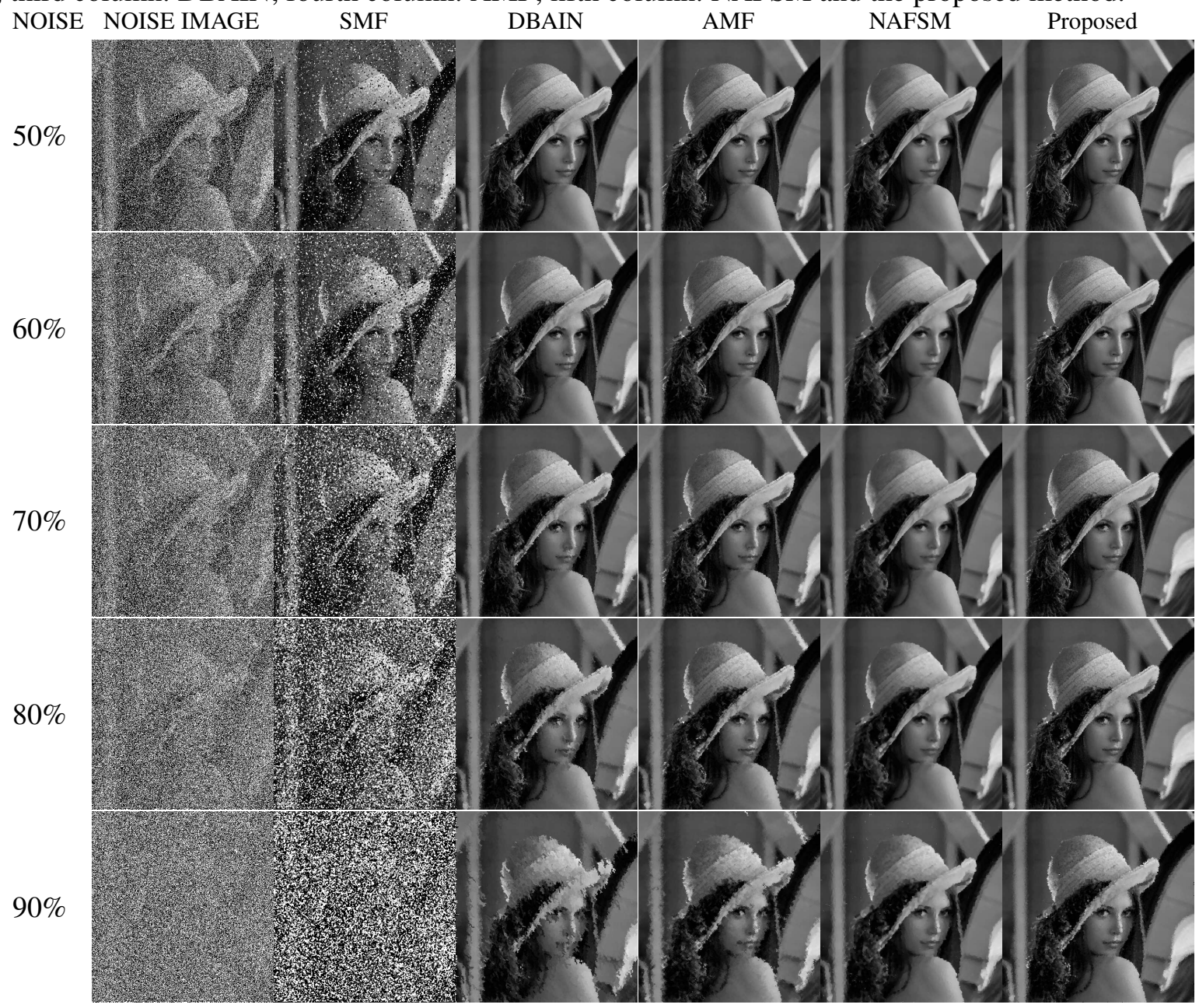


Table 4: SSIM values for diferents filters applied on images of figure 7 at noise density levels of $40 \%$ to $90 \%$. The best results were highlighted.

\begin{tabular}{|c|c|c|c|c|c|c|c|c|c|c|}
\hline \multirow[t]{2}{*}{ Filters } & SMF & DBAIN & $\mathrm{AMF}$ & NAFSM & Proposed & SMF & DBAIN & $\mathrm{AMF}$ & NAFSM & Proposed \\
\hline & \multicolumn{5}{|c|}{$40 \%$} & \multicolumn{5}{|c|}{$50 \%$} \\
\hline Baboon & 0.580 & 0.934 & 0.929 & 0.913 & 0.939 & 0.375 & 0.893 & 0.897 & 0.871 & 0.918 \\
\hline Barbara & 0.467 & 0.882 & 0.854 & 0.892 & 0.833 & 0.283 & 0.823 & 0.806 & 0.851 & 0.793 \\
\hline Cameraman & 0.492 & 0.984 & 0.985 & 0.975 & 0.987 & 0.280 & 0.971 & 0.975 & 0.963 & 0.983 \\
\hline Einstein & 0.469 & 0.901 & 0.881 & 0.893 & 0.874 & 0.270 & 0.856 & 0.846 & 0.861 & 0.845 \\
\hline Hill & 0.469 & 0.876 & 0.854 & 0.859 & 0.849 & 0.265 & 0.822 & 0.808 & 0.820 & 0.818 \\
\hline House & 0.494 & 0.99 & 0.991 & 0.986 & 0.992 & 0.271 & 0.980 & 0.984 & 0.979 & 0.990 \\
\hline Jet plane & 0.527 & 0.981 & 0.981 & 0.975 & 0.983 & 0.321 & 0.965 & 0.969 & 0.964 & 0.977 \\
\hline Lake & 0.584 & 0.973 & 0.972 & 0.964 & 0.977 & 0.380 & 0.952 & 0.958 & 0.949 & 0.969 \\
\hline Ship & 0.432 & 0.867 & 0.841 & 0.857 & 0.844 & 0.266 & 0.812 & 0.801 & 0.816 & 0.808 \\
\hline Walk bridge & 0.620 & 0.945 & 0.941 & 0.931 & 0.950 & 0.423 & 0.911 & 0.917 & 0.901 & 0.934 \\
\hline Average & 0.513 & 0.933 & $\begin{array}{r}0.923 \\
60 \%\end{array}$ & 0.925 & 0.923 & 0.313 & 0.898 & $\begin{array}{r}0.896 \\
70 \%\end{array}$ & 0.898 & 0.903 \\
\hline Baboon & 0.222 & 0.829 & 0.849 & 0.821 & 0.888 & 0.375 & 0.893 & 0.897 & 0.871 & 0.918 \\
\hline Barbara & 0.160 & 0.758 & 0.754 & 0.811 & 0.756 & 0.283 & 0.823 & 0.806 & 0.851 & 0.793 \\
\hline Cameraman & 0.162 & 0.946 & 0.960 & 0.950 & 0.976 & 0.280 & 0.971 & 0.975 & 0.963 & 0.983 \\
\hline Einstein & 0.139 & 0.799 & 0.798 & 0.825 & 0.810 & 0.270 & 0.856 & 0.846 & 0.861 & 0.845 \\
\hline Hill & 0.131 & 0.742 & 0.746 & 0.768 & 0.778 & 0.265 & 0.822 & 0.808 & 0.820 & 0.818 \\
\hline House & 0.149 & 0.961 & 0.974 & 0.971 & 0.986 & 0.271 & 0.980 & 0.984 & 0.979 & 0.990 \\
\hline Jet plane & 0.192 & 0.936 & 0.954 & 0.950 & 0.970 & 0.321 & 0.965 & 0.969 & 0.964 & 0.977 \\
\hline Lake & 0.235 & 0.920 & 0.936 & 0.928 & 0.957 & 0.380 & 0.952 & 0.958 & 0.949 & 0.969 \\
\hline Ship & 0.144 & 0.745 & 0.742 & 0.765 & 0.768 & 0.266 & 0.812 & 0.801 & 0.816 & 0.808 \\
\hline Walk bridge & 0.250 & 0.855 & 0.877 & 0.865 & 0.912 & 0.423 & 0.911 & 0.917 & 0.901 & 0.934 \\
\hline Average & 0.178 & 0.849 & $\begin{array}{r}0.859 \\
80 \%\end{array}$ & 0.865 & 0.880 & 0.313 & 0.898 & $\begin{array}{r}0.896 \\
90 \%\end{array}$ & 0.898 & 0.903 \\
\hline Baboon & 0.118 & 0.735 & 0.776 & 0.756 & 0.845 & 0.375 & 0.893 & 0.897 & 0.871 & 0.918 \\
\hline Barbara & 0.087 & 0.662 & 0.681 & 0.761 & 0.704 & 0.283 & 0.823 & 0.806 & 0.851 & 0.793 \\
\hline Cameraman & 0.100 & 0.906 & 0.937 & 0.932 & 0.965 & 0.280 & 0.971 & 0.975 & 0.963 & 0.983 \\
\hline Einstein & 0.068 & 0.719 & 0.730 & 0.778 & 0.763 & 0.270 & 0.856 & 0.846 & 0.861 & 0.845 \\
\hline Hill & 0.065 & 0.660 & 0.675 & 0.709 & 0.727 & 0.265 & 0.822 & 0.808 & 0.820 & 0.818 \\
\hline House & 0.088 & 0.926 & 0.956 & 0.958 & 0.978 & 0.271 & 0.980 & 0.984 & 0.979 & 0.990 \\
\hline Jet plane & 0.113 & 0.888 & 0.927 & 0.930 & 0.956 & 0.321 & 0.965 & 0.969 & 0.964 & 0.977 \\
\hline Lake & 0.144 & 0.861 & 0.904 & 0.905 & 0.941 & 0.380 & 0.952 & 0.958 & 0.949 & 0.969 \\
\hline Ship & 0.071 & 0.641 & 0.659 & 0.706 & 0.714 & 0.266 & 0.812 & 0.801 & 0.816 & 0.808 \\
\hline Walk bridge & 0.145 & 0.762 & 0.822 & 0.818 & 0.879 & 0.423 & 0.911 & 0.917 & 0.901 & 0.934 \\
\hline Average & 0.100 & 0.776 & 0.807 & 0.825 & 0.847 & 0.313 & 0.898 & 0.896 & 0.898 & 0.903 \\
\hline
\end{tabular}

\section{CONCLUSIONS}

The classic morphological filters are effective tools for the removing of impulsive noise, however as the noise level increases they are ineffective. This is because the structuring element is rigid and does not fit to the local characteristics of the image. On the other hand, the adaptive morphological filters used to eliminate impulsive noise are more effective than the classic morphological filters. However, the imposition of the property of adjunction makes complicated a definition of the structural elements so that the operators erosion and dilation do not modify the noisy pixels, coupled with the fact that they are not reversible operators, results in operators that are not very effective at high impulsive noise levels. In order not to alter non-noisy pixels, it is proposed a pair of operations $\gamma_{R}$ and $\varphi_{R}$ that turn out to be morphological filters, $\wedge$-filters and $\vee$-filters which led to define morphological openings and closings. These characteristics make the filters effective in eliminating high levels of impulsive noise while preserving fine details of the objects in the image and obtaining comparable results with other state-of-the-art methods, even exceeding them with respect to the time-quality ratio.

\section{REFERENCES}

Ćurić V, Landström A, Thurley MJ, Hendriks CL (2014). Adaptive mathematical morphology-a survey of the field. Pattern Recognit Lett 47:1828. https://doi.org/10.1016/j.patrec.2014.02.022.

Debayle J, Pinoli JC (2005). Spatially adaptive 
morphological image filtering using intrinsic structuring elements. Image Anal Stereol 24(3):145-58. https://www.iasiss.org/ojs/IAS/article/view/782.

Debayle J, Pinoli JC (2005). Adaptive-Neighborhood Mathematical Morphology and its Applications to Image Filtering and Segmentation. In 9th European Congress on Stereology and Image Analysis (ECSIA) 2:123-30. https://ieeexplore.ieee.org/document/1530447.

Debayle J, Pinoli JC (2009). General Adaptive Neighborhood Mathematical Morphology. In IEEE International Conference on Image Processing (ICIP): 2249-52. https://https://ieeexplore.iee.org/document/5413979.

Angulo J, Velesco-Forero S. (2011). Structurally Adaptive Mathematical Morphology Based on Nonlinear Scale-Space Decompositions. Image Anal Stereol 30(2): 111-22. https://www.iasiss.org/ojs/IAS/article/view/892.

Legaz-Aparicio AG, Verdú-Monedero R, Angulo J (2018). Adaptive morphological filters based on a multiple orientation vector field dependent on image local features. J Comput Appl Math 330: 965-81. https://doi.org/10.1016/j.cam.2017.05.001.

Huang T, Yang G, Tang G (1979). "A fast twodimensional median filtering algorithm". IEEE Trans Acoust Speech, Signal Process 27(1):13-18. https://ieeexplore.ieee.org/abstract/document/11631

Ibrahim H, Kong NSP, Ng TF (2008). Simple adaptive median filter for the removal of impulse noise from highly corrupted images. IEEE Trans Consum Electron 54(4):1920-27. https://ieeexplore.ieee.org/document/4711254.

Maragos P, Schafer R (1987). Morphological filtersPart I: Their set-theoretic analysis and relations to linear shift-invariant filters. IEEE Trans Acoust Speech, Signal Process 35(8):1153-69. https://ieeexplore.ieee.org/document/1165259.

Mukhopadhyay S, Chanda B (2002). An edge preserving noise smoothing technique using multiscale morphology. Signal Process
82(4):527-44. https://doi.org/10.1016/S01651684(01)00143-8.

Oh J, Chaparro LF (1998). Adaptive fuzzy morphological filtering of images. In Proceedings of the 1998 IEEE International Conference on Acoustics, Speech and Signal Processing, ICASSP'98 (Cat. No. 98CH36181). 5: 2901-04. https://ieeexplore.ieee.org/document/678132/

Serra J (1983). Image analysis and mathematical morphology. Academic Press.

Serra J, Vicent L (1992). An overview of morphological filtering. Circ, Syst Signal Pr 11(1):47-108. https://doi.org/10.1007/BF01189221.

9. Song J, Delp EJ (1990). The analysis of morphological filters with multiple structuring elements. Computer Vision, Graphics, and Image Processing 50(3):308-28. https://doi.org/10.1016/0734189X(90)90150-T.

Srinivasan KS, Ebenezer D (2007). A new fast and efficient decision-based algorithm for removal of high-density impulse noises. IEEE Signal Proc Let 14(3):189-92. https://ieeexplore.ieee.org/document/4100656.

Toh KKV, and Mat Isa NA (2010). Noise Adaptive Fuzzy Switching Median Filter for Salt-and-Pepper Noise Reduction. IEEE Signal Proc Let, 17(3): 281-84. https://ieeexplore.ieee.org/document/5356178.

8.Wang Z, Zhang D (1999). Progressive switching median filter for the removal of impulse noise from highly corrupted images. IEEE $\mathrm{T}$ Circuits-II 46(1):78-80. https://ieeexplore.ieee.org/document/749102.

Wang Z, Bovik AC, Sheikh HR, Simoncelli EP (2004). Image quality assessment: from error visibility to structural similarity. IEEE T Image Process 13(4): 600-12. https://ieeexplore.ieee.org/document/1284395.

Zhao Y, Li D, Li Z (2007). Performance enhancement and analysis of an adaptive median filter. In 2007 Second International Conference on Communications and Networking in China 65153. https://ieeexplore.ieee.org/document/4469475. 